\title{
La representación cognitiva del mapa electoral
}

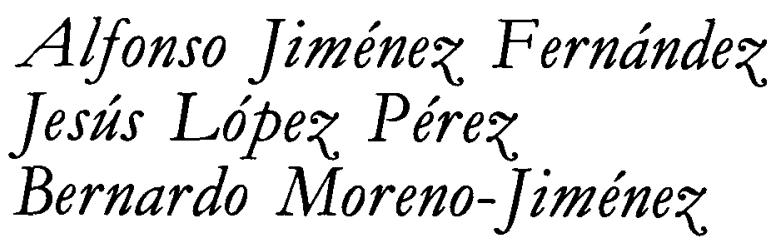

Universidad Autónoma de Madrid

\section{INTRODUCCION}

La percepción sociopolítica no es un área nueva de estudio, sino más bien un tema de reciente y renovado desarrollo. El auge del paradigma cognitivo en Psicologia Social ha facilitado su incremento y extensión, la aparición de las técnicas multivariadas ha permitido un nuevo y más útil tratamiento de los datos, y al desarrollo de un amplio software estadístico ha permitido afrontar una cantidad ingente de datos, inaccesibles de otra forma. La conjunción de los tres elementos hace actualmente viable investigaciones anteriormente irrealizables, especialmente aquellas que dentro de un marco cognitivo multiplican los datos y la intersección de éstos.

Así, por ejemplo, en el área especifica que nos ocupa, la percepción sociopolítica, Messick (1961) utilizando el análisis multidimensional, estudió la percepción de los líderes políticos, estableciendo que su percepción podia ser representada en un espacio de siete dimensiones. Posteriormente trabajos sobre el mismo tema realizados por Ahrems (1967) en
Alemania, Sherman y Ross (1972) en USA, Forgas, Kagan y Frey (1977) en Inglaterra, mostraron que era posible su representación en un número menor de dimensiones, y que tres dimensiones podían bastar para su representación. Tales autores mostraron que todas las dimensiones variaban en función de la cultura nacional, aunque la dimensión evaluativa parecia reproducirse en las diferentes investigaciones. Un estudio con las mismas técnicas MDS ha podido comprobar cómo la orientación política no parecía afectar fundamentalmente el número de dimensiones necesarias para su representación, pero sí la identidad de éstas. Recientemente Tetlock (1983) ha estudiado la relación entre los estilos cognitivos como variable de personalidad y la ideologia política.

Otras técnicas han mostrado igualmente su utilidad para estudiar la percepción socio-política: el Diferencial Semántico, el Análisis Factorial, el Análisis de Varianza, y en general los métodos multivariados. Igualmente la metodología y los recursos procedentes del área de los registros psicofisiológicos han 


\section{0}

mostrado su utilidad y valor en este ámbito (Jiménez, Moreno y López, 1983).

La Psicología de los Constructos (Kelly, 1955) ha ofrecido un marco de referencia fecundo para el estudio del ámbito político. Su enfasis en la percepción personal de la realidad y su correspondiente "construcción del mundo" en un articulado jerárquico de constructos, ha originado una corriente de investigación que desde la perspectiva cognitiva ha alcanzado la casi totalidad de los comportamientos humanos (Stringer-Bannister, 1979; Mancuso-Adams Webber, 1982).

La metodologia principal de investigación se ha centrado en el instrumento ideado por Kelly (1955) el RCPT (Role Construct Repertory Test o Rep Grid) que permite cuantificar el mundo cognitivo del sujeto. Aunque su ámbito de aplicación original fue la Psicologia Clínica su utilización se ha ampliado a una extensa gama de comportamientos que van desde la percepción estética hasta el mercado automovilístico (Fransella-Bannister 1977; Slater, 1976; Rivas y Marco, 1985).

La utilización del Rep Grid en el ámbito político ha sido también extensa, aunque la mayoría de los estudios se han desarrollado dentro del marco conceptual de la teoria de los Constructos Personales. Warr, Schroder y Blackman (1979) lo han utilizado para el análisis político, Mihevc (1978) para el estudio de la valencia afectiva y la cantidad de información poseída acerca de los líderes políticos. Soucer y Du Cette (1971) lo han utilizado para el estudio de las diferencias sexuales en la discriminación política, Gaugler y Zalking (1975) lo han hecho aplicándolo al análisis de los debates políticos.

Sin embargo el Rep Grid no parece haberse utilizado para el estudio de la conducta electoral, cuando por sus características estructurales y sus posibilidades de tratamiento parece un instrumento idóneo para analizar la "construcción política» que el individuo se hace de los lí- deres de los Partidos Políticos, de los lemas políticos y en general de los diversos elementos del ámbito político, en una situación electoral que se presenta intencionalmente como una reestructuración de la percepción y las actitudes políticas.

En función de la exposición anterior nuestra investigación parte de dos presupuestos básicos:

1. El Rep Grid puede ser utilizado como un instrumento para el análisis de la percepción político-electoral.

2. La información gráfica obtenida por el Rep Grid puede ser considerada como una aproximación a la representación mental del sujeto respecto a la estructuración de la situación político-electoral.

Para ello es necesario la elaboración de un tipo de rejilla político-electoral que permita contrastar y discriminar los estímulos políticos. Las formas que este tipo de rejilla puede adoptar son muy amplias y dependerá de los objetivos específicos de la investigación.

Dados estos presupuestos introductorios el objeto de la presente investigación es doble:

1. Describir a partir del Rep Grid el "Universo politico" que un sujeto o una población se ha construido a partir del material suministrado por la propaganda electoral icónica.

2. Desarrollar las metodologías de análisis más adecuadas al objetivo anterior. En la fase actual de la investigación nos centraremos en la fase descriptiva de los datos.

\section{METODO}

\section{Sujetos}

Dado el carácter descriptivo de esta fase la investigación no se procedió a un control de las variables de la muestra y 
se atendió únicamente a obtener una muestra que incluyera jóvenes, jóvenes adultos y adultos. La muestra estuvo integrada por cien sujetos: 35 estudiantes de 3. ${ }^{\circ}$ de BUP, 46 estudiantes Universitarios y 19 amas de casa.

\section{Instrumentos}

Para obtener la descripción del «Universo Político» a partir de la información electoral suministrada por la propaganda icónica con ocasión de las elecciones municipales de 1983, se elaboraron cuatro modelos de rejilla político-electoral que parecían cubrir el espectro político de aquel momento y que están recogidas en las tablas 1-4.

TABLA 1

Rejilla de los lemas electorales generales (filas) y Partidos Politicos (columnas).

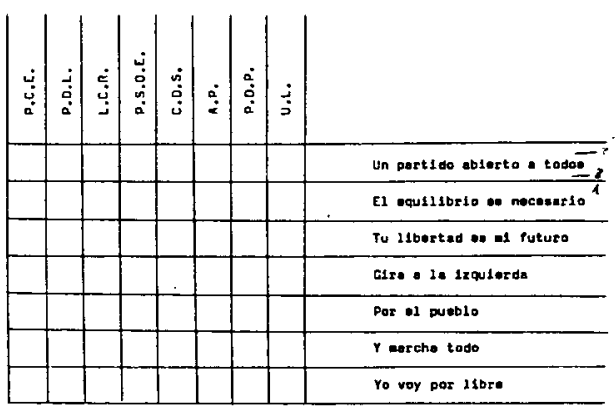

TABLA 2

Rejilla de los lemas electorales generales (filas) y lideres nacionales de los Partidos Políticos (columnas).

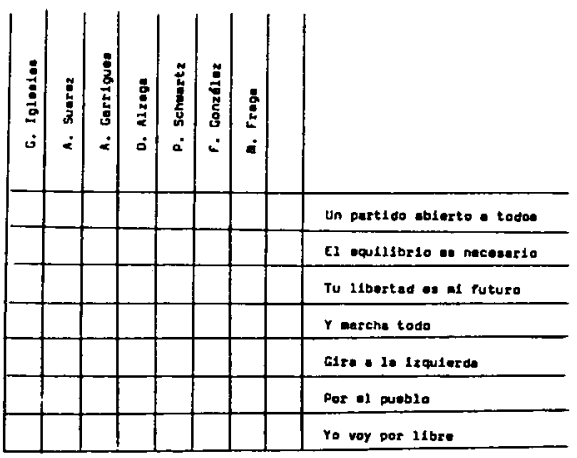

TABLA 3

Rejilla de los lemas electorales municipales (filas) y los candidatos a alcalde de los Partidos Politicos (columnas).

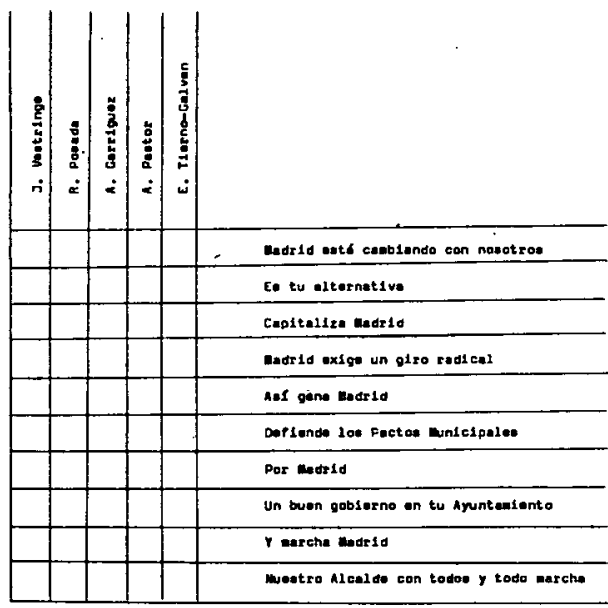

TABLA 4

Rejilla de los lemas electorales autonómicos (filas) y los candidatos a Presidente de la Autonomía de Madrid de los Partidos Políticos (columnas).

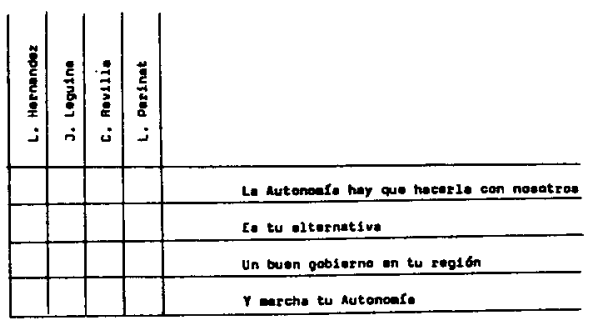

\section{Procedimiento}

Los sujetos cumplimentaron las cuatro rejillas propuestas grupalmente. Se formaron tres grupos, estudiantes de $3 .^{\circ}$ de BUP, estudiantes universitarios y amas de casa a los que se les dieron las instrucciones verbalmente al mismo tiempo que podían proceder a leerlas. Las instrucciones fueron leidas una sola vez $y$ aplicadas de idéntica manera a las 4 rejillas. En ellas se les pedia a los sujetos que determinaran en una escala de 1 a 5 
el grado de conveniencia de los lemas electorales a los Partidos Políticos o a sus líderes respectivos.

\section{Análisis}

Para el análisis de los datos se eligieron cuatro sistemas de análisis de los habitualmente utilizados con la rejilla de Kelly: la medida de intensidad relacional, el análisis de los parámetros de las columnas y las filas, el análisis factorial de componentes principales, y el análisis de conglomerados. La elección de estos cuatro sistemas de análisis fue debida al presupuesto de que, conjuntamente, podrian describir y representar el «Universo Politicon buscado.

La medida de intensidad relacional (Bannister, 1960, 1962) ha sido ampliamente utilizada en el área de la Psicología de los Constructos, principalmente, como medida de integración conceptual. Esta medida es, fundamentalmente, un indice correlacional obtenido a partir de la elevación al cuadrado de todas las correlaciones de un mismo constructo y su multiplicación por cien. La suma de todos los resultados de los constructos es considerada como la intensidad relacional total. La diferencia de los índices relacionales permite establecer una jerarquía entre los diferentes constructos. Fransella y Bannister (1977) han desarrollado una técnica que permite representar gráficamente los resultados a través de un eje de coordenadas. El eje horizontal representa el elemento principal - el de mayor indice de intensidad relacional-, y el eje vertical representa el elemento secundario - aquél con mayor intensidad relacional sin que correlacione significativamente con el constructo principal一. En definitiva la intensidad relacional permite obtener el elenco jerárquico de los constructos presentes, la integración total entre ellos y la gráfica correspondiente.

Aunque la medida de intensidad relacional es un tipo de medida relativamente sencilla, permite obtener resultados muy semejantes a los obtenidos con téc- nicas multivariadas. Según FransellaBannister (1977) el constructo principal obtenido con este procedimiento coincide con el primer factor obtenido con el análisis factorial de componentes principales. Igualmente la resolución gráfica se aproxima a los resultados obtenidos con el análisis de conglomerados.

En el análisis de los parámetros de las columnas y las filas se han desarrollado dos tipos de puntuaciones. Una es la Puntuación Global (P.G.) que consiste en la suma y media respectiva de los constructos, es decir, de los lemas. La otra puntuación, la Puntuación Total (P.T.) es la suma y media respectiva de los elementos, es decir, de los Partidos Políticos. Ambas puntuaciones son totalmente descriptivas y no suponen ningún análisis inferencial de los datos.

La puntuación P. G. indica la aplicabilidad del lema a los diferentes Partidos Políticos. A partir de este tipo de puntuación es posible obtener un "Indice de Conveniencia» de cada lema con su Partido Político. Este índice vendría dado por los siguientes elementos: propio.

- Máxima puntuación en su lema

- P. G. del lema inferior a la media. Es decir que el lema sería poco aplicable a los restantes Partidos Políticos.

La puntuación P. T. puede proporcionar un "Indice de la amplitud" ideológico-política del Partido. Teóricamente cabe esperar que esta amplitud sea mayor en los Partidos Políticos de mayor amplitud ideológica, probablemente Partidos del Centro, y menor en los Partidos Políticos más radicales.

Rivas (1981) considera el análisis factorial como la metodologia más actual de las utilizadas con la rejilla. Aunque la afirmación no es unánimemente acepta$\mathrm{da}$, es probable que sea la metodología más extendida, Slater (1977) ha desarrollado el programa «Ingrid» para diversos tratamientos factoriales de los datos ( $\mathrm{Ri}$ vas, 1985). Recientemente Keen y Bell 


\section{Estudios}

(1980) han presentado su programa "Griddle» al que consideran de más fácil manejo. El método de análisis factorial de componentes principales nos ha parecido especialmente útil para el análisis de las dimensiones subyacentes en los diversos sloganes o lemas electorales.

Su aplicación nos ha parecido adecuada sólo para el análisis de los lemas, pero no para el análisis de los Partidos Políticos o líderes. Teóricamente su aplicación debe permitirnos conocer los factores ideológicos subyacentes en los diferentes sloganes politicos utilizados por la totalidad de los Partidos Politicos. El número de factores encontrados deberia depender no del número de lemas presentes en la campaña, sino de su polarización politico-ideológica. Esto podría suponer que en unas Elecciones Generales el número de dimensiones encontradas será mayor que en unas Elecciones Municipales.

Diversos autores han criticado el análisis factorial como el más indicado para el análisis de la rejilla y han propuesto como alternativa el análisis de conglomerados (Goodge, 1979; Shaw, 1980, 1981; Thomas y Harri-Augstein, 1985). Sin entrar en la discusión técnica, en nuestra investigación el análisis de conglomerados se ha utilizado para obtener los agrupamientos entre las variables cualitativas que son los Partidos Políticos. Como Cuadras (1981) lo define, este tipo de análisis permite obtener el grado de similaridad entre determinadas variables. Nuestra intención era obtener la similaridad de los Partidos Políticos a partir de la distancia ultramétrica ofrecida por el dendograma. De esta forma puede obtenerse una visión general de las afinidades político-electorales y una apreciación de la percepción de los grandes bloques politicos en una determinada campaña electoral.

Los diferentes análisis aludidos se realizaron con el ordenador VAX-11/780 con sistema operativo VMS mediante la introducción de los datos en su terminal VT-131 y la salida de resultados se realizó con una impresora LA-100.

\section{3}

\section{RESULTADOS}

Dada la extensión de los resultados obtenidos y del carácter prospectivo del presente estudio se exponen a continuación únicamente los resultados obtenidos a partir de la tabla 5. El cuadro 1 expone la matriz final de datos. A partir de ella se han obtenido los restantes resultados.

\section{TABLA 4}

Rejilla media de los 100 sujetos con los lemas electorales generales (filas) y Partidos Politicos (columnas).

\begin{tabular}{|c|c|c|c|c|c|c|c|c|}
\hline بُ بُ & $\begin{array}{l}\dot{a} \\
\dot{0} \\
\dot{a}\end{array}$ & نّ & 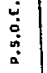 & $\begin{array}{l}\dot{0} \\
\dot{0} \\
\dot{0}\end{array}$ & $\vdots$ & $\begin{array}{l}a^{*} \\
a \\
a \\
a\end{array}$ & تُ & \\
\hline 3,02 & 2,59 & 2,40 & 3,83 & 2,89 & 2,18 & 2,40 & 2,54 & Un pertido goinert o a todan \\
\hline 2,51 & 2,82 & 2,20 & 3,34 & 3,24 & 2,56 & 2,66 & 2,68 & El equilibria an neceoserio \\
\hline 3,09 & 7,58 & 2,79 & 3,63 & 2,90 & 2,23 & 2,41 & 2,50 & Tu lloneted on al futuro \\
\hline 4,09 & 2.30 & 4,01 & 3,79 & 2,34 & 1,57 & 1,81 & $2, \overline{06}$ & Gles e in lequier de \\
\hline 5,51 & 2,57 & 3,21 & 3,43 & 2,69 & 2,13 & 2,24 & 2.37 & Dof al audoto \\
\hline 2,59 & 2,56 & 2,33 & 3,19 & 2,76 & 2,55 & 2,55 & 2,70 & $Y$ ereche todo \\
\hline 2.24 & 3,69 & 2,73 & 2,40 & 2,47 & 2,25 & 2,44 & 2,71 & Yo voy gos $11 \mathrm{Bre}$ \\
\hline
\end{tabular}

\section{Análisis de los lemas}

La tabla 6 muestra los resultados de cada Partido Político en su propio lema. La mínima puntuación posible es 1 y la

\section{TABLA 6}

Indices de Conveniencia

\begin{tabular}{lcc}
\cline { 2 - 3 } & \multicolumn{3}{c}{$\begin{array}{c}\text { Media } \\
\text { obtenida } \\
\text { por el } \\
\text { proplo } \\
\text { partido }\end{array}$} & $\begin{array}{c}\text { Media } \\
\text { para } \\
\text { partidos los }\end{array}$ \\
\hline Lemas & 3,02 & 2,68 \\
\hline $\begin{array}{l}\text { Un Partido abierto a todos } \\
\text { (PCE) }\end{array}$ & 2,56 & 2,80 \\
$\begin{array}{l}\text { Tu libertad es mi futuro } \\
\text { (P.D.L.) }\end{array}$ & 2,69 & 2,46 \\
$\begin{array}{l}\text { Yo voy por libre } \\
\text { (P.D.L.) }\end{array}$ & 4,01 & 2,56 \\
$\begin{array}{l}\text { Gira a la izquierda } \\
\text { (L.C.R.) }\end{array}$ & 3,83 & 2,68 \\
$\begin{array}{l}\text { Por el pueblo } \\
\text { (PSOE) }\end{array}$ & 3,24 & 2,69 \\
$\begin{array}{l}\text { El equilibrio es necesario } \\
\text { (CDS) }\end{array}$ & 2,95 & 2,69 \\
$\begin{array}{l}\text { Y marcha todo } \\
\text { (AP) }\end{array}$ & 2,65 & 2,72 \\
$\begin{array}{l}\text { Y marcha todo } \\
\text { (P.D.P.) }\end{array}$ & 2,70 & 2,72 \\
$\begin{array}{l}\text { Y marcha todo } \\
\text { (U.L.) }\end{array}$ & & \\
\hline
\end{tabular}


máxima 5. A continuación aparece la media del lema aplicado a los restantes partidos. Ambos resultados, conjuntamente, proporcionan el «Indice de conveniencia» del lema.

La inspección conjunta de las tablas 5 y 6 permite el análisis de la idoneidad del lema. En primer lugar, la observación de la puntuación P. L. permite apreciar la asociación del lema al Propio Partido Político. La observación de la tabla 5 permite un análisis diferencial de la aplicabilidad de los lemas. Este tipo de análisis permite una primera aproximación a la ideología percibida de los Partidos Políticos.

La tabla 7 indica el orden jerárquico de los lemas, obtenido a partir de la medida de intensidad relacional. El orden jerárquico indica el nivel de asociación de un lema con todos los demás. Dentro de la Teoría de los constructos el primer constructo, en este caso lema, es llamado "constructo superordinado", es decir, aquel del cual dependen los demás. Igualmente puede identificarse con lo que Asch (1946) llamaba «rasgo central». $\mathrm{El}$ orden jerárquico puede ser asociado a lo que Rivas (1981) ha denominado "constructos referentes claves».

\section{TABLA 7}

Ordenación jerárquica de los lemas. Resultado obtenido mediante la medida de intensidad relacional

Jerarquia relacional de las lemas

\begin{tabular}{lll}
\hline $1 .^{\circ}$ & Tu libertad es mi futuro & 385,88 \\
$2 .^{\circ}$ & Un Partido abierto a todos & 379,82 \\
$3 .^{\circ}$ Por el pueblo & 352,27 \\
$4 .^{\circ}$ Gira a la izquierda & 291,55 \\
$5 .^{\circ}$ El equilibrio es necesario & 244,06 \\
$6 .^{\circ}$ Y marcha todo & 217,09 \\
$7 .^{\circ}$ Yo voy por libre & 131,19 \\
\hline
\end{tabular}

Los cuatro primeros lemas tienen una correlación positiva y significativa entre ellos. El último de los lemas tiene una correlación de $-0,09$ con el primero. Aunque ambos aluden a la libertad han sido percibidos de forma claramente diferente. Es probable que el orden jerár- quico de los lemas coincida, o al menos se aproxime, al orden de valores prevalentes para el elector.

La figura 1 muestra las distancias espaciales entre los lemas a partir de las coordenadas determinadas por el lema principal: «Tu libertad es mi futuro», y el lema secundario: «El equilibrio es necesario".

FIGURA 1

Medida de intensidad relacional de los lemas

Representación espacial

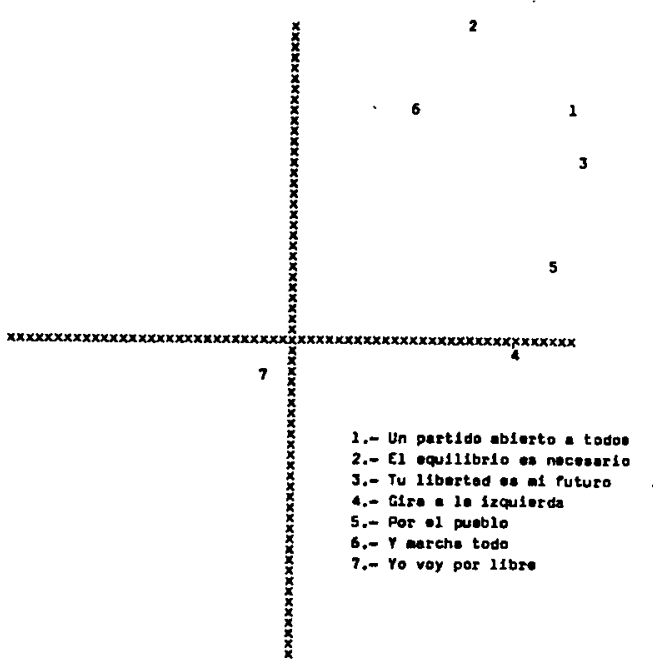

El gráfico muestra una relativa proximidad entre los lemas 1 y 3 , índice de que en función de ambos ejes, ambos lemas indican conceptos muy próximos. Se puede observar igualmente que hay dos lemas claramente diferenciados de los demás: "Gira a la izquierda" y "Yo voy por libre».

Un análisis complementario de las correlaciones muestra que los lemas $1,3,5$, 2 y 6 correlacionan todos positivamente entre sí. El lema 4 correlaciona negativamente con los lemas 2 y 6 . El lema 7 correlaciona negativamente con los lemas $1,2,3,5$ y 6 . Estos dos lemas, 4 y 7 , claramente diferentes de los restantes, 
tienen entre ellos una correlación prácticamente nula $(r=0,01)$.

La tabla 8 presenta los resultados del análisis factorial de componentes principales con rotación varimax.

TABLA 8

Análisis factorias de los lemas Rotación Varimax

\begin{tabular}{crrrr}
\hline & \multicolumn{4}{c}{ Factor } \\
\cline { 2 - 5 } Lema & \multicolumn{1}{c}{$\mathbf{1}$} & \multicolumn{1}{c}{$\mathbf{2}$} & \multicolumn{1}{c}{$\mathbf{4}$} \\
\hline 1 & 0,751 & 0,611 & $-0,070$ & 0,215 \\
2 & 0,111 & 0,942 & $-0,060$ & 0,302 \\
3 & 0,877 & 0,441 & $-0,002$ & 0,181 \\
4 & 0,980 & $-0,129$ & 0,016 & $-0,141$ \\
5 & 0,988 & 0,123 & $-0,072$ & 0,033 \\
6 & 0,016 & 0,470 & $-0,312$ & 0,825 \\
7 & $-0,037$ & $-0,059$ & 0,981 & $-0,180$ \\
Varianza & ex- & & & \\
plicada & 3,283 & 1,711 & 1,074 & 0,905 \\
\hline
\end{tabular}

Cuatro factores explican el 99,62 por 100 de la varianza total. El primer factor es claramente el más importante, con una varianza total explicada del 59,19 por 100. Es un factor en el que saturan altamente los lemas $5,4,3$ y 1 . Se refiere a los valores relativos a los intereses del pueblo, los valores de la libertad, la participación de todos y el giro a la izquierda. Es un factor unipolar que puede ser categorizado como «Izquierda Democrátican.

El segundo factor está marcado por la alta saturación del segundo lema: «El equilibrio es necesarion. La alta saturación de los lemas 3, 2 y 6 matizan y aclaran el sentido del factor. Es un factor unipolar que puede ser denominado «Equilibrio aperturista».

El tercer factor está representado casi exclusivamente por el lema «Yo voy por libre». En el polo negativo satura el lema n. 6: "Y marcha todo». Indica un factor bipolar caracterizado en un poco por la indiferencia y despreocupación y en el otro por la eficacia y organización.

El cuarto factor, con una aportación muy escasa a la varianza total (3 por 100) está representado principalmente por el lema 6: "Y marcha todo" y en menor grado por el lema 2: «El equilibrio es necesario". Es un factor unipolar de «Eficacia Política» o "Tecnocracia».

\section{Análisis de los partidos políticos}

La tabla 9 muestra los resultados que permiten apreciar la amplitud ideológica de los diferentes Partidos Políticos.

La Puntuación Total (P. T.) se obtiene por la suma de las puntuaciones obtenidas en todos los lemas. La Puntuación Media (P. M.) indica la media respectiva. Se parte del presupuesto que la mayor puntuación $\mathrm{P}$. $T$. indica la amplitud de la cobertura ideológica del partido. Una apreciación más matizada puede obtenerse atendiendo a la puntuación media en los lemas que representan el eje principal y secundario de la medida de intensidad relacional.

TABLA 9

Cobertura ideológica de los partidos

Puntuaciones medias obtenidas en los lemas electorales

\begin{tabular}{lcccc}
\hline & $\begin{array}{c}\text { Puntuación } \\
\text { Global }\end{array}$ & $\begin{array}{c}\text { Puntuación } \\
\text { Medla }\end{array}$ & $\begin{array}{c}\text { Media } \\
\text { 1. }\end{array}$ & $\begin{array}{c}\text { Media } \\
\mathbf{2}^{\circ} \text { Eje }\end{array}$ \\
\hline P.C.E. & 21,05 & 3,03 & 3,45 & 2,48 \\
P.D.L. & 18,09 & 2,58 & 2,50 & 2,69 \\
L.C.R. & 19,75 & 2,82 & 3,12 & 2,42 \\
P.S.O.E. & 23,85 & 3,40 & 3,72 & 2,99 \\
C.D.S. & 19,22 & 2,74 & 2,70 & 2,82 \\
A.P. & 15,87 & 2,26 & 2,02 & 2,59 \\
P.D.P. & 16,69 & 2,38 & 2,23 & 2,68 \\
U.L. & 17,66 & 2,52 & 2,39 & 2,69 \\
\hline
\end{tabular}


La figura 2 representa las similaridades entre los Partidos Políticos. Se ha obtenido a partir de la medida de intensidad relacional.

\section{FIGURA 2}

Representación bidimensional de los partidos políticos a los que hacen referencia los carteles estudiados a partir de su medida de intensidad relacional

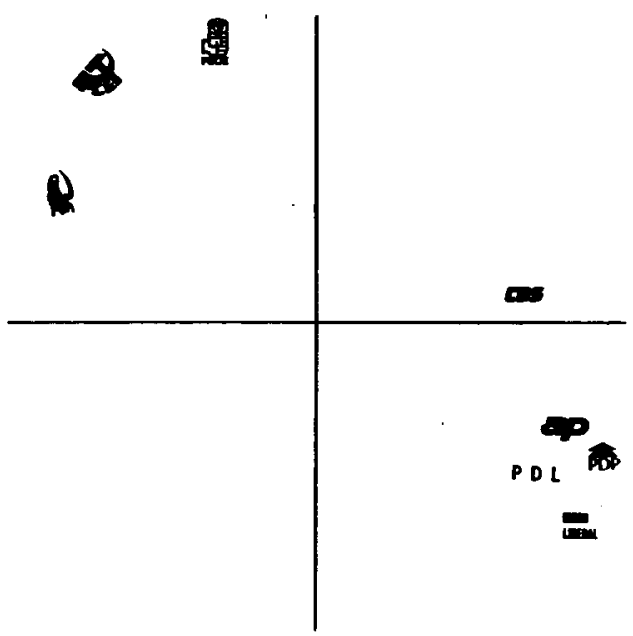

El gráfico muestra claramente la existencia de tres grupos de Partidos Políticos situándolos en tres cuadrantes diferentes. En el primer cuadrante se sitúan PCE, PSOE y LCR. En el segundo cuadrante, aislado, aparece el CDS. En el cuarto cuadrante, opuesto al primero, se sitúan AP, PDP, PDL y UL.

Un análisis más detallado indica que los Partidos del primer cuadrante correlacionan todos entre sí positiva y significativamente ( $\mathrm{p}<0,05)$. Lo mismo ocurre con todos los Partidos del cuarto cuadrante.

La figura 3 muestra el dendograma del análisis de conglomerados de los Partidos Políticos.

El análisis de conglomerados es efectuado utilizando el programa $1 \mathrm{M}$ del BMDP. De las diferentes modalidades
FIGURA 3

Agrupación de los partidos politicos a los que hacen referencia los carteles estudiados mediante análisis de Cluster

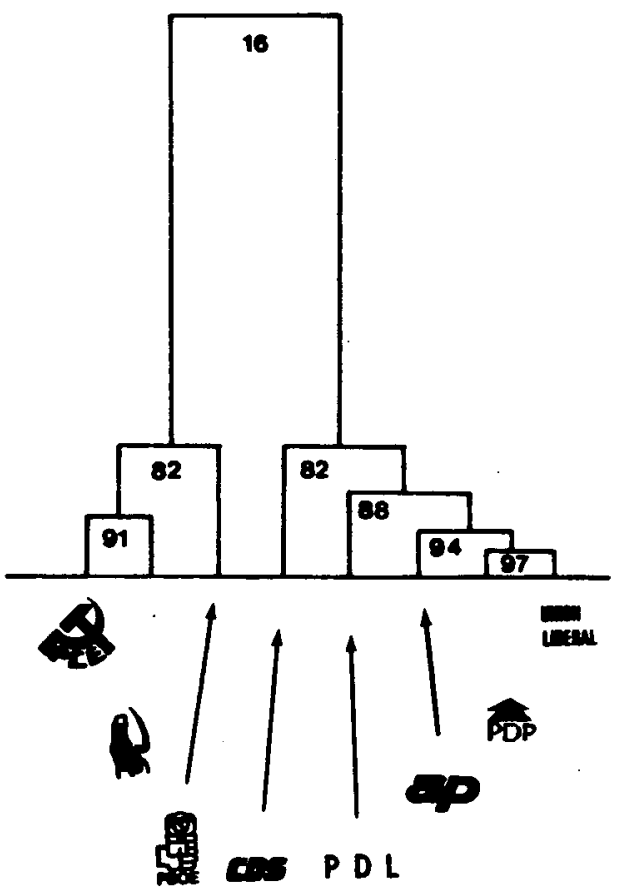

posibles se ha elegido el sistema CORRAVE. Este sistema procede a formar los conglomerados teniendo en cuenta el signo de la correlación y promediando las distancias para formar un nuevo conglomerado.

Como puede observarse se han obtenido tres conglomerados principales. Por una parte, a la izquierda, LCR, PCE y PSOE, por otra, a la derecha, PDL, AP, PDP y UL. En el Centro, aunque vinculado al último conglomerado, aparece el CDS.

\section{CONCLUSIONES}

La ausencia de control de las variables incidentes en la muestra ha hecho de nuestro trabajo una elaboración de las posibilidades metodológicas del Rep Grid en el análisis de la propaganda político-electoral. 
La propuesta aquí realizada desarrolla una novedad metodológica no explorada ni sistematizada anteriormente.

Como resultado, las conclusiones no pueden ser sino de orden metodológico, aunque, secundariamente, puedan también derivarse inferencias sobre la estructura percibida de la situación políticoelectoral.

Sería conveniente la utilización de técnicas complementarias para el análisis de los datos. Dos tipos de análisis parecen especialmente adecuados: El Análisis Multidimensional y el Análisis Factorial de Correspondencias. El control de las variables de la muestra permitiría la aplicación de técnicas inferenciales.
A partir de los análisis anteriores, obtenido el mapa cognitivo político-electoral, probablemente seria factible la localización de la posible ubicación politica del sujeto. Igualmente deberia estudiarse las posibilidades de predicción de la conducta electoral del sujeto a partir de su topografia cognitiva de la situación electoral. Los resultados obtenidos en algunos de los análisis efectuados y los resultados de las Elecciones Municipales de 1983 en la Provincia de Madrid, auguran la viabilidad del proyecto y su posible aplicación en futuras campañas electorales.

\section{Referencias}

Adams-Webblir, J. R.: Personal Construct. London, Wiley, 1979.

AsCH, S. E.: "Forming impressions of personality". Journal of Abnormal an Social Psychology, 1946, 41, 258290.

AHrFNs, H. J.: «Zur Systimatik der Urteibsdildung beider Beurteilung westdeuttscher Politiken». Arcbiven fur die gisante Psycbologie, 1967, 119, 57-89.

BANNISTER, D.: "Conceptual structure in thougth disordered schizophrenic». Journal of Mental Science, 1960, 106, 1230-1249.

BANNISTER, D.: "The nature and the measurement of schizophrenic thought disorder». Journal of Mental Science, 1962, 108, 825-842.

BIERI, J: «Cognitive complexity-Simplicity and predictive behavior». Journal of Abnormal and Social Psychology, 1955, 51, 263-268.

BIER̊, J.: «Cognitive Complexity and personality development». En O. J. Harvey (Ed) Experience, Structure and adaptability. N. Y. Springer 1966.

CuAdRAS, C. M.: Métodos de análisis multivariante. Barcelona: Editorial Universitaria de Barcelona, 1981.

Forgas, J. P.; KagAN, C. M., y Frey, D.: «The cognitive representation of political personalities: a cross-cultural comparaison." International Journal of Psychology, 1977, 12, 19-30.

FranselLA, F., y BANNISTER, D.: "A validation of repertory grid technique as a measure of political consţruing». Acta Psycbologica, 1967, 97-106.

Franshilla, F., y Bannister, D.: A Manual for repertory grid technique. N. Y. Academic Press, 1977.

Gaugker, E. A. Y Zalkind, S. S.: “Dimensions of civil liberties and Personality: relationship for measures of tolerance for complexity». Journal of Social Issues. 1975, 31, 93-110.

GOODGE, P.: «Problems of repertory grid analyses and a cluster analysis solution». British Journal Psycholo$g y, 1979,134,516-521$.

Herman, H.: Análisis Factorial Moderno. Madrid, Saltés, 1980.

JIMENEZ, A.; MORENO-JIMENEZ, B., y LOPEZ, J.: «Actividad electrodérmica como sistema de evaluación de los estimulos icónico-electorales». En III Encuentro Nacional de Psicologia Social, Las Palmas, 1983.

KEEN, T. R., Y BELL, R.: "One thing leads to another: A new approach to elicitation in the repertory grid techniquem. International Journal of Man-Machine Studies, Spetial Edition, Sep, 1980.

KelLLY, G.: The Psychology of Personal Construct. N. Y. Norton, 1955.

LANDFIFLD, A. W.: "Grid relationship scoring used with a Rep test modification", Psychological Reports, $1967,21,19$.

LANDFiEld, A. W.: Personal Construct Systems in Psychotherapy. Rand McNally, 1971.

Mancuso, J. C., y Adams-Webber, J.: The Construing Person. N. Y. Praeger, 1982.

Messick, S.: "The perceived structure of political relationship". Sociometry, 1961, 24, 270-278.

MinEvC, N. T.: «Information, valence, and cognitive complexity in the political domain», The Journal of Psychology, 1978, 99, 163-177.

RATHOD, P.: «Methods for the analysis of rep grid data». En H. Bonarius, R. Holland, y S. Rosemberg, (Ed). Personal Construct Psychology, N. Y. S. Martin Press, 1981. 
Rivas, F.: "La rejilla como técnica psicométrica de medida de la ejecución típica individual». Analisis $y$ Modificación de Conducta, 1981, 15, 171-246.

Rivas, F., y MARco, R.: Evaluación conductual subjetiva: la técnica de la rejilla. Centro Editorial de Servicios y Publicaciones Universitarias. Valencia, 1985.

SEAMAN, J. M., y KonNig F.: «A comparison of measures of cognitive complexity». Sociometry, 1974, 37, 375-390.

ScotT, W. A.: "Cognitive Structure». Theory and measurement of Individual differences. Whashington, V. H. Winston, 1979.

SHAw, M. L. G.: On becoming a personal scientis. N. Y. Academic Press, 1980.

SHAW, M. L. G.: Recent advances in Personal Construct Tecbnology. N. Y. Academic Press, 1981.

SHERMAN, R. E., y ROSS, L. B.: «Liberalism-conservatism and dimensional salience in the perception of political figures". Journal of Personality and Social Psychology, 1972, 23, 120-127.

SLATER, P.: Explorations of intrapersonal Space. Vol 1. London, Wiley, 1976.

SLATHR, P.: Dimensions of intrapersonal space, Vol 2., London Wiley, 1977.

SOUCAR, E., Y Du CETTE, J.: "Cognitive complexity and political preference». Psychological Reports, 1971, $29,373-374$.

Stringer, P., Y BAnnister, D.: Constructs of sociality and individuality. N. Y. Academic Press, 1980.

Tetlock, PH. E.: «Cognitive Style and Political ideology». Journal of Personality and Social Psichology, 1983, 45, 118-126.

Thomas, L. F., y Hari-Augstein, F. S.: Self-Organised learning. London, Routledge, 1985.

VAN DERD KLOOT, W. A.: «Multidimensional scaling of repertory grid responses: two aplications of Homals». En H. Bonarius, A. Holland y S. Rosemberg (Ed). Personal Construct Psychology. N. Y. San Martin Prees, 1981.

WARr, P. H. M.; SROder, HaMA., y Blackman, S.: "The structure of political judgement». British Journal" of Social and Clinical Psychology, 1969, 8, 32-43. 